\title{
Trends in cardiovascular disease: Are we winning the war?
}

\author{
Simon Capewell DSc, Martin O'Flaherty MD
}

$\infty \infty$

\author{
See related research paper by Tu and colleagues, page E118
}

ardiovascular disease generates a substantial burden of illness in Canada and beyond. Yet recent epidemiologic trends have been very encouraging. Deaths and rates of morbidity from cardiovascular disease fell by at least 50\% in most countries from about 1980 to 2000. Some two-thirds of this decline can be attributed to a decrease in adverse events and reflects reductions in the prevalence of major risk factors. The remaining third is attributable to reduced case-fatality rates, owing mainly to treatments. ${ }^{1}$ This victory is worth celebrating; but are we winning our long-term war against cardiovascular disease?

In this issue, Tu and colleagues report on trends in cardiovascular disease in Canada between 1994 and 2004. ${ }^{2}$ Mortality due to cardiovascular disease fell by $30 \%$ in this period. The decline was slightly more for acute myocardial infarction than for stroke and heart failure. Hospitalization rates for stroke and heart failure fell by $27 \%$, whereas age-adjusted hosital admission rates for acute myocardial infarction apparently fell by only $9 \%$. The true proportion is probably greater but concealed by the effects of the aging and growth of the population, by diagnostic changes that have inflated patient numbers and by the counting of episodes rather than of patients. The practice by hospitals of recoding diagnoses to maximize income may also have contributed to the apparent $33 \%$ decline of in-hospital case-fatality rates among patients with acute myocardial infarction. In contrast, reductions in case-fatality rates due to stroke and heart failure were relatively modest. An increase in the average age of patients in Canada, as elsewhere, was consistent with a "compression of morbidity," which means that disease commences at a later age and affects a shorter period of the total life span. It is crucial to note that most deaths occurred outside of hospital, many among individuals with no prior diagnosis of cardiovascular disease.

As the first such evaluation in Canada, the study by Tu and colleagues had many strengths. It was comprehensive, specific to Canada and based on data of adequate quality. Its acknowledged limitations included its analysis of episodes rather than individuals, leaving open the possibility that a greater reduction in incident-related hospitalizations may have occurred but been obscured by an increase in recurrent admissions. A lack of information on case mix means that severity of disease may have declined as thresholds for admission to hospital were relaxed. The apparent decline in case-fatality rates may thus have been inflated.

The findings of Tu and colleagues are generally consistent with those of analyses in the United States and Europe. Using linked data in Scotland, McIntyre and colleagues ${ }^{3}$ found that admissions for chest pain and unstable angina increased by

\section{Key points}

- In Canada between 1994 and 2004, mortality and rates of hospitalization due to cardiovascular disease declined by about $30 \%$.

- Reductions in the prevalence of major risk factors contributed to about two-thirds of this decline, whereas the remainder is attributable to treatments.

- Over $80 \%$ of cases of premature cardiovascular disease can be prevented through population-wide control of tobacco and governmental policies that promote a healthy diet.

about $40 \%$ while rates of myocardial infarction declined by about $30 \%$. In the Netherlands, a study by Koek and colleagues ${ }^{4}$ using a similar approach found that rates of myocardial infarction declined by $20 \%$.

Such studies provide valuable information for quantifying trends in burden of disease and making cautious projections. It is problematic, however, to assume that rates of hospitalization represent an accurate measure of incidence or that they can be used to estimate prevalence directly. Drawing a relation between trends in mortality and hospital admission and changes in medical and surgical treatments is also complex. Furthermore, many out-of-hospital deaths among patients with no prior diagnosis of cardiovascular disease are sudden, and thus amenable only to primary prevention.

Canadians see themselves as being different from Americans. Trends in cardiovascular disease over the last 3 decades, however, show a striking similarity. Ford and colleagues ${ }^{5}$ recently reported a $43 \%$ total decline in deaths due to coronary heart disease between 1980 and 2000. Using a validated and comprehensive policy model, they attributed about $47 \%$ of this reduction to specific medical therapies. Among these therapies, revascularization made a surprisingly small contribution. A decrease in the prevalence of major risk factors potentially contributed to two-thirds of the total decline in mortality. However, this improvement was offset by substantial increases in rates of obesity and diabetes, which were in turn compounded by persistence in the prevalence of smoking (particularly among younger age groups) and rates of hypertension that were previously in decline but have now, ominously flattened. ${ }^{5}$ These adverse trends in serious risk factors for cardiovascular disease are worrying, especially given recent similar trends in many other countries. Analyses of Canadian data will thus be of great interest.

Is the party over? Flattening rates of death from cardiovascular disease in younger age groups have now been reported

Simon Capewell and Martin O'Flaherty are with the Division of Public Health, University of Liverpool, United Kingdom. 
in the United States, the United Kingdom, Australia and elsewhere. This stall in progress is occurring in spite of the availability of evidence-based therapies for the majority of eligible patients. ${ }^{6.7}$ Worsening trends in cardiovascular disease may be even greater among people in socially deprived groups. ${ }^{8}$ Given that rates of cardiovascular disease increase steeply with age, demographic aging compounded by an expansion in population in most industrialized countries represents an iceberg of hidden cardiovascular disease which will become visible in the very near future.

What are the implications of these trends for clinicians and health care systems in Canada and elsewhere? Cardiovascular disease will remain the most common cause of death for the near future. Large numbers of patients with cardiovascular disease will flood our hospitals. Over time they will be older and thus more challenging to treat. Therapeutic extension of life expectancy will be correspondingly shorter, forcing us to concentrate more on improving quality of life rather than just prolonging it slightly.

Prevention, therefore, becomes vital, because over $80 \%$ of premature cardiovascular disease is avoidable. ${ }^{9}$ Medications to reduce lipids and blood pressure will help. But the promotion of population-wide control of tobacco, cessation of smoking, a healthier diet and increased physical activity is crucial. A large impact could be made by such interventions at the global, national and provincial levels, potentially halving the future burden of cardiovascular disease. ${ }^{10,11}$ Feasible policy, legislative and fiscal measures could eradicate industrial trans fats, halve the dietary intake of saturated fats and salt, make fresh fruit and vegetables cheaper, increase physical activity and render smoking prohibitively expensive. Healthcare professionals have a major responsibility to support such initiatives, which could substantially reduce the costly societal burden of cardiovascular disease. However, tracking the effectiveness of preventive interventions will demand regular, high-quality monitoring. We therefore look to $\mathrm{Tu}$ and colleagues from Statistics Canada and to other sources to provide us with reports on a regular basis. We have triumphed in recent battles against cardiovascular disease but we need to win the longer war.

\section{Competing interests: None declared.}

Contributors: Both of the authors conceived the ideas for this manuscript and both drafted and approved the final version of the manuscript submitted for publication. Simon Capewell is the guarantor of the manuscript.

\section{REFERENCES}

1. Tunstall-Pedoe H, Kuulasmaa K, Mähönen M, et al. Contribution of trends in survival and coronary-event rates to changes in coronary heart disease mortality: 10year results from 37 WHO MONICA project populations. Monitoring trends and determinants in cardiovascular disease. Lancet 1999;353:1547-57.

2. Tu JV, Nardi L, Fang J. National trends in rates of death and hospital admissions related to acute myocardial infarction, heart failure and stroke, 1994-2004. CMAJ 2009;180:E118-25.

3. MacIntyre K, Murphy NF, Chalmers J, et al. Hospital burden of suspected acute coronary syndromes: recent trends. Heart 2006;92:691-2.

4. Koek HL, de Bruin A, Gast A, et al. Decline in incidence of hospitalisation for acute myocardial infarction in the Netherlands from 1995 to 2000. Heart 2006;92:162-5.

5. Ford ES, Ajani UA, Croft JB, et al. Explaining the decrease in US deaths from coronary disease, 1980-2000. N Engl J Med 2007;356:2388-98.

6. Ford ES, Capewell S. Coronary heart disease mortality among young adults in the US from 1980 through 2002: concealed leveling of mortality rates. J Am Coll Cardiol 2007;50:2128-32.

7. O'Flaherty M, Ford E, Allender S, et al. Coronary heart disease trends in England and Wales from 1984 to 2004: concealed levelling of mortality rates among young adults. Heart 2008;94:178-81.

8. O'Flaherty M, Bishop J, Redpath A, et al. Recent leveling of coronary heart disease mortality rates among young adults in Scotland may reflect major social inequalities: a time trend study. BMJ. In press.

9. Yusuf S, Hawken S, Ônpuu S, et al. Effect of potentially modifiable risk factors associated with myocardial infarction in 52 countries (the INTERHEART study): case-control study. Lancet 2004;364:937-52.

10. Aslan BU, Critchley JA, Capewell S. Small changes in United Kingdom cardiovascular risk factors could halve coronary heart disease mortality. J Clin Epidemiol 2005;58:733-40. Epub 2005 Apr. 18.

11. Asaria P, Chisholm D, Mathers C, et al. Chronic disease prevention: health effects and financial costs of strategies to reduce salt intake and control tobacco use. Lancet 2007;370:2044-53.

Correspondence to: Prof. Simon Capewell, Division of Public Health, University of Liverpool, Liverpool L69 3GB, United Kingdom; fax 0044151794 5588; capewell@liverpool.ac.uk 